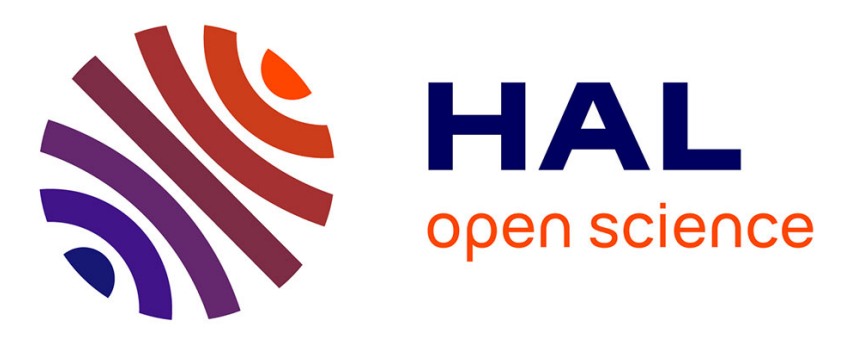

\title{
Getting hold of approaching objects: In search of a common control of hand-closure initiation in catching and grasping
}

Cornelis van de Kamp, Raoul M. Bongers, Frank T.J.M. Zaal

\section{To cite this version:}

Cornelis van de Kamp, Raoul M. Bongers, Frank T.J.M. Zaal. Getting hold of approaching objects: In search of a common control of hand-closure initiation in catching and grasping. Human Movement Science, 2010, 29 (4), pp.518. 10.1016/j.humov.2010.02.010 . hal-00659888

\section{HAL Id: hal-00659888 https://hal.science/hal-00659888}

Submitted on 14 Jan 2012

HAL is a multi-disciplinary open access archive for the deposit and dissemination of scientific research documents, whether they are published or not. The documents may come from teaching and research institutions in France or abroad, or from public or private research centers.
L'archive ouverte pluridisciplinaire HAL, est destinée au dépôt et à la diffusion de documents scientifiques de niveau recherche, publiés ou non, émanant des établissements d'enseignement et de recherche français ou étrangers, des laboratoires publics ou privés. 


\section{Accepted Manuscript}

Getting hold of approaching objects: In search of a common control of handclosure initiation in catching and grasping

Cornelis van de Kamp, Raoul M. Bongers, Frank T.J.M. Zaal

PII:

S0167-9457(10)00050-3

DOI:

10.1016/j.humov.2010.02.010

Reference:

HUMOV 1223

To appear in:

Human Movement Science

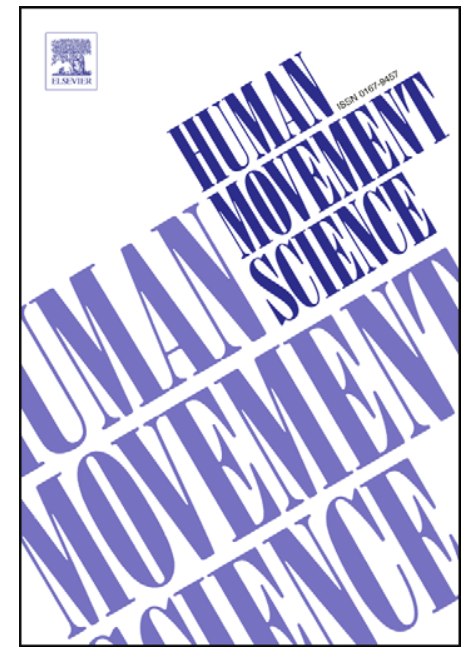

Please cite this article as: van de Kamp, C., Bongers, R.M., Zaal, F.T.J., Getting hold of approaching objects: In search of a common control of hand-closure initiation in catching and grasping, Human Movement Science (2010), doi: 10.1016/j.humov.2010.02.010

This is a PDF file of an unedited manuscript that has been accepted for publication. As a service to our customers we are providing this early version of the manuscript. The manuscript will undergo copyediting, typesetting, and review of the resulting proof before it is published in its final form. Please note that during the production process errors may be discovered which could affect the content, and all legal disclaimers that apply to the journal pertain. 
Getting hold of approaching objects: In search of a common control of handclosure initiation in catching and grasping

Cornelis van de Kamp, Raoul M. Bongers, \& Frank T. J. M. Zaal ${ }^{*}$

Center for Human Movement Sciences

University Medical Center Groningen, Sector F

University of Groningen

P. O. Box 196

NL-9700 AD Groningen

The Netherlands

* Corresponding author

e-mail: f.t.j.m.zaal@rug.nl

phone: $\quad+31503637717$

fax: $\quad+31503633150$ 


\section{Abstract}

Both in the catching and grasping component of prehension, the hand opens and closes before hand-object contact is made. The initiation of hand closure has to be coordinated with the time course of the decrease of the distance between the hand and the target object, i.e., with the reaching component in prehension or the approach of the target in catching. The authors investigated if this initiation of hand closure could be explained by a common control. For this purpose, they fitted the dynamic timing model to data from the two tasks. In both tasks, participants were asked to get hold of an object approaching along the table top at a constant velocity. In the prehension task, participants could reach out to grasp the object; in the catching task, they were required to keep their hand stationary. In comparison with other accounts, the dynamic timing model performed best in explaining the data. The model proved adequate for the prehension task but not for the current catching task.

Psychlnfo Classification 2300

Keywords: prehension time-to-contact perception-action dynamic systems motor control 


\section{Introduction}

Prehension, the act of coordinated reaching and grasping, and catching have in common that the hand first opens and subsequently closes to take hold of the target object. In prehension, the initiation of the hand closure has to be coordinated with the reaching (i.e., the movement of the hand towards the target). In catching, the initiation of hand closure has to be coordinated with the arrival of the approaching target. Thus, in both tasks, the gap between the hand and the target closes, and the timing of the initiation of closure of the hand has to be coordinated with this gap closure. The objective of the current study was to see if the control of catching and the control of the grasping component of prehension could be understood within the same framework. More specifically, given the success of the dynamic timing model in the context of prehension (Zaal \& Bootsma, 2004; Zaal, Bootsma, \& van Wieringen, 1998), in which first-order time-to-contact information is used to time the initiation of hand closure, we focused on this model to see if it would predict this timing both in the grasping of prehension and in catching equally well.

Two decades of prehension research offered several proposals that described how the initiation of hand closure might be timed. Unfortunately, most of these proposals did not hold very well under experimental scrutiny. Three of these proposals will be briefly presented in the following. In the first proposal, hand closure was thought to be initiated at a fixed time before hand-object contact, independent of task conditions (Gentilucci, Chieffi, Scarpa, \& Castiello, 1992). Several empirical studies tested this hypothesis by manipulating reaching amplitude (Zaal et al., 1998), object width (Zaal \& Bootsma, 2004), object size 
(Wang \& Stelmach, 2001), object orientation (Rand \& Stelmach, 2005), reach or object velocity (Carnahan \& McFadyen, 1996; Rand, Squire, \& Stelmach, 2006; Zaal et al., 1998), and the direction of the object's approach (Watson \& Jakobson, 1997), which, taken together, demonstrated that in prehension the initiation of hand closure is not time-invariant over a range of conditions.

A second proposal employed a spatial rather than a temporal variable: The initiation of hand closure should happen at a fixed distance from the hand to the target object (cf. Wang \& Stelmach, 1998, 2001). Recently, Stelmach and colleagues have amended this original proposal (Rand et al., 2006; Rand, Shimansky, Hossain, \& Stelmach, 2008), after finding that closing distance varied with object orientation (Rand \& Stelmach, 2005) and reach velocity (Rand et al., 2006). They proposed that the initiation of hand closure occurs at a closing distance that is a function of, among other variables, reaching amplitude and velocity. Although adding these variables improved the data fitting, it made the account less parsimonious, of course.

A third proposal that we will discuss stems from the line of research that considers the timing of interceptive actions to be based on prospective information about the time remaining until the object and the observer meet (e.g., Bootsma \& Peper, 1992; Lee, 1976; Savelsbergh, Whiting, \& Bootsma, 1991). In this proposal, the initiation of hand closure is triggered on the basis of first-order time-to-contact information (tau). In prehension, this first-order time-to-contact information specifies the time for the physical gap between the target object and the grasping hand to be closed under prevailing speed conditions. One way the initiation of hand closure could be based on first-order time-to-contact information is that the hand starts closing at a critical value of tau. In prehension, however, this seems not to be the case. For instance, earlier work showed that at the initiation of hand closure, first- 
order time-to-contact varied with reaching amplitude (Zaal \& Bootsma, 2004; Zaal et al., 1998) and object velocity (Zaal et al., 1998). However, Zaal and coworkers (Zaal \& Bootsma, 2004; Zaal et al., 1998) showed another way first-order time-tocontact information could be at the basis of the initiation of hand closure, along the lines of Schöner's (1994) dynamic timing model. We will return to this model later, after having had a look at the catching literature.

As in the literature on prehension, also in the context of catching, researchers have considered the proposal that the initiation of hand closure is timed on the basis of a critical value of first-order time-to-contact. Just as in prehension, little support was found for this proposal. That is to say, ambiguous results concerning values of first-order time-to-contact at the initiation of hand closure were found in studies on catching. Whereas an early study reported no effects of object velocity (Savelsbergh, Whiting, Burden, \& Bartlett, 1992), later studies showed that firstorder time-to-contact at the initiation of hand closure varied with object velocity (Bennett, van der Kamp, Savelsbergh, \& Davids, 1999; Caljouw, van der Kamp, \& Savelsbergh, 2004; Wallace, Stevenson, Weeks, \& Kelso, 1992). Also, just as was the case for prehension, it was examined whether the initiation of hand closure is timed on the basis of a critical closing time or closing distance. Findings concerning these hypotheses were inconsistent as well (Laurent, Montagne, \& Savelsbergh, 1994; Mazyn, Montagne, Savelsbergh, \& Lenoir, 2006; Savelsbergh et al., 1992; Wallace et al., 1992; Wang \& Stelmach, 2001).

Together, the proposals introduced so far, do not seem very promising in revealing the control for the separate tasks, let alone for an account for the timing of hand closure common in both prehension and catching. Therefore, we turn to a final proposal, one that has been shown to accurately predict the moment of hand closure in prehension (Zaal \& Bootsma, 2004; Zaal et al., 1998) and might apply to 
catching as well. In the same vein as the critical-tau (threshold) type of control mentioned before, the dynamic timing model exploits first-order time-to-contact information. However, in this account, the use of tau is more sophisticated than simply waiting until a critical value has been reached. The idea is that grasping behavior is best understood within a dynamical systems approach, in which the hand-opening and hand-closing states are endowed with stability features.

Elaborating on the work of Schöner (1994), Zaal and colleagues $(1998,2004)$ proposed a formulation of how the stability of the hand-opening state and the stability of the hand-closing state are coupled to first-order time-to-contact. At the beginning of the movement, when first-order time-to-contact between the grasping hand and the target object is long, the hand-opening state is most stable, whereas the hand-closing state is rather unstable. While the reach unfolds, first-order timeto-contact decreases, resulting in a loss of stability of the hand-opening state and a gain of stability of the hand-closing state. At a certain point in time (i.e., the initiation of hand closure), the hand-closing state has become more stable than the handopening state and a transition - which has stability features of its own - from the hand-opening state to the hand-closing state takes place (for more details see Schöner, 1994; Zaal \& Bootsma, 2004; Zaal et al., 1998). A consequence of this nonlinear dynamics alternative for the use of first-order time-to-contact over the threshold type of control is that hand closure does not necessarily occur at a fixed value of first-order time-to-contact. That is to say, in the situation of non-constant velocities, whereas values of first-order time-to-contact at the moment of the initiation of hand closure might vary across experimental conditions, the dynamic timing model might be able to accommodate these variations (cf. Zaal \& Bootsma, 2004; Zaal et al., 1998). Furthermore, the dynamical regulation of the initiation of 
hand closure makes the system resistant to perturbations, for instance, when the hand needs to be retracted or the target object changes position.

Zaal and Bootsma (2004) considered the standard prehension task of having participants pick up stationary target objects, and showed that closing time, closing distance, and first-order time-to-contact at the moment of hand-closure initiation all varied with factors such as distance and the size of the objects, but no effects were seen when looking at the differences between the model predictions and the actual moments of hand-closure initiation. These differences had been computed on a trial-to-trial basis. In an earlier study, Zaal and colleagues (1998) had studied the picking up of objects that either remained stationary or moved away from the participants in the experiments. They inspected the performance of the dynamic timing model, using compound time-to-contact time series rather than individual ones, and showed that the model predicted the moments of initiation of hand closure quite accurately. Here, we will present an experiment in which participants were asked to either reach for and pick up an approaching target (prehension task) or keep their hand still and wait for the object to arrive into the hand that they needed to open and close to get hold of the approaching object (catching task). Following Zaal and Bootsma (2004), we will evaluate the data on a trial-to-trial basis, to investigate if the dynamic timing model also applies to the situation of approaching objects, both in prehension and in catching.

\section{Method}

\subsection{Participants}

Seven men and eight women, with an average age of 24 years (ranging from 20 to 41 years) participated in the experiment. All were right-handed and had normal or corrected-to-normal vision. Participants were naive to the exact purpose of the 
experiment, gave their informed consent and were paid a small fee for their participation.

\subsection{Apparatus}

A cylindrical target object was placed on top of a magnet embedded objectcarrier. This carrier was made to move along a plain white tabletop $(2 \mathrm{~m} \times 2 \mathrm{~m})$ by means of a magnetic coupling to a servomotor driven mechanism underneath the tabletop (for a similar setup, see: Schenk et al., 2000). The exact movement path of the target object was computer-controlled through a user interface that was developed for this purpose (LabView, National Instruments). The positions of four infrared light emitting diodes (IREDs) were tracked at a rate of $100 \mathrm{~Hz}$ using an Optotrak system (NDI, Waterloo, Ontario, Canada). The IREDs were placed on the center of the target object, the lateral lower corner of the index finger nail, the medial lower corner of the thumb nail, and the skin immediately proximal to the styloid process of the radius at the wrist.

\subsection{Procedure and design}

We used two tasks (prehension and catching) in which the target object (diameter: $3 \mathrm{~cm}$, height: $1.5 \mathrm{~cm}$ ) approached the participants with one of three constant velocities $(20,40$ and $60 \mathrm{~cm} / \mathrm{s})$ and starting from one of two initial distances $(75$ and $100 \mathrm{~cm})$. With a set acceleration of $600 \mathrm{~cm} / \mathrm{s}^{2}$ it took the object at most $6 \mathrm{~cm}(100 \mathrm{~ms})$ to reach the constant velocity. Participants sat alongside the table, with their right side touching the table edge and their sagittal plane parallel to the table edge. The object approached along the participants' sagittal plane, some $30 \mathrm{~cm}$ away from the edge of the table. At the start of each trial, the right hand, with the tips of the thumb and index finger touching, was positioned on a Plexiglas span; this span allowed the object to pass underneath. In the prehension task, 
participants were to reach for and grasp the object. As soon as the object started to move towards the participant, he or she was free to choose the moment to start the reach to grasp movement. This way, the pick-up location was left to the participant; the only instruction was to carry out a continuous, fast but accurate reaching-tograsp movement. In the catching task, the participants were required to catch the approaching object between the pads of the thumb and index finger while keeping the position of their hand fixed, resting on the Plexiglas span. After liftoff, the object was to be placed on the tabletop somewhere around the pickup location. During each trial headphone-delivered white noise was played.

The order of the catching and prehension tasks was counterbalanced across participants. Presenting the 6 randomized conditions ( 3 object velocities $\times 2$ initial distances) in 12 blocks for the two tasks resulted in a total of 144 trials per participant.

\subsection{Data analysis}

A total of 2080 trials were used for the data analysis. In 3 trials the object was unintentionally dropped, while in 46 trials, either some IRED data was missing or we encountered problems with the object carrier.

Position data was smoothed using a low-pass recursive Butterworth filter with a cutoff frequency of $10 \mathrm{~Hz}$. Speed was computed using a three-point finite difference algorithm. Hand position was defined as the average position of the thumb and index-finger IRED. The start of the reaching movement was defined as the moment at which the tangential hand speed rose above a threshold of $2 \mathrm{~cm} / \mathrm{s}$. Hand aperture was defined as the three-dimensional distance between the thumb and the index finger IREDs. The start and end of the grasping movement were defined as 
the moment when hand opening and closing speed rose above or dropped below a threshold of $2 \mathrm{~cm} / \mathrm{s}$, respectively.

To determine the initiation of hand closure we looked back from the moment of peak closing velocity and detected the first moment that the hand closing speed passed a threshold of $2 \mathrm{~cm} / \mathrm{s}$. Closing distance was defined as the distance (projected along the dimension of the object approach) between the object and the hand at the initiation of hand closure. Closing time was the time from the initiation of hand closure until the end of the grasping movement. First-order time-to-contact ${ }^{1}$ $T C_{1}(D)$ at the moment of hand-closure initiation corresponded to the time it would take to make contact with the object if conditions would prevail (i.e., constant velocity) and was computed by dividing the distance (projected along the dimension of the object approach) between the object and the hand by the momentary speed at which this distance was closed.

An important part of our analyses examined the accuracy with which the initiation of hand closure was predicted by the dynamic timing model (Schöner, 1994; Zaal \& Bootsma, 2004; Zaal et al., 1998). For each trial, we compared the predicted moment of hand-closure initiation with the experimentally observed moment of hand-closure initiation. To arrive at the model prediction, we numerically simulated the model's set of differential equations (see Appendix) using a RungeKutta algorithm, with a fixed time step of $0.01 \mathrm{~s}$, equal to the time step of the kinematic data. All parameters of the model were set at a fixed value $(\alpha=10 ; \omega=$ 10; $\gamma=10 ; \beta=90 ; \sigma=0.75 ; r_{\text {crit }}=0$ ) except the parameter $c_{\text {vision, }}$ which was allowed to vary across participants and tasks (as discussed later). The parameter $c_{\text {vision }}$ represents the strength of the contribution of the first-order time-to-contact variable (see Appendix; cf. Schöner, 1994; Zaal \& Bootsma, 2004; Zaal et al., 1998). We optimized the values of the $c_{\text {vision }}$ parameter to have a best fit between the average 
model predictions and the average observed initiation moments by finding the $c_{v i s i o n}$ parameter setting that resulted in a minimum sum of squared prediction errors. In a first pass, we allowed $c_{\text {vision }}$ to vary only across participants (see Table 1 for the values of $\left.c_{\text {vision }}\right)$. In a subsequent analysis, $c_{\text {vision }}$ was allowed to vary across participants but also between tasks (prehension vs. catching; see Table 1).

\section{${ }^{* * *}$ Table 1 about here ${ }^{* * *}$}

Model accuracy was evaluated in terms of a temporal prediction error, which was defined as the time difference of the experimentally observed moment of handclosure initiation and the moment of hand-closure initiation as predicted by the dynamic timing model; a positive difference meant that the model prediction preceded the observed moment of initiation of hand closure.

A problem for the comparison of the four dependent variables that we identified before (closing distance, closing time, first-order time-to-contact, and dynamictiming-model prediction error) is that they are defined along different dimensions. To arrive at dependent measures that are defined along the same dimension for each dependent variable, we computed temporal prediction errors, analogous to the dynamic-timing-model prediction error, for each variable that we considered in our comparison. To this end, we assumed that these variables (closing time, closing distance, or first-order time-to-contact) were to be kept constant at a specific value in the control of grasping. We took this value (which was allowed to vary across participants) to be the value at which the sum of squared prediction errors was minimal, just as we had done for the dynamic-timing-model error. Next we inspected for each trial when this value was reached, and computed the difference in time between the latter moment and the moment of hand-closure initiation. We did so for the variables of closing time, closing distance, and firstorder time-to-contact. The resulting dependent variables were all along the 
dimension of real time, just as the prediction error of the dynamic timing model. Thus, a fair comparison among all four of the temporal prediction errors was possible.

Each dependent variable (temporal prediction errors of closing distance, closing time, first-order time-to-contact, and the dynamic timing model) was analyzed with a separate repeated-measures analyses of variance (ANOVAs) with task (catching vs. prehension), object velocity $(20,40$, or $60 \mathrm{~cm} / \mathrm{s})$, and initial distance (75 vs. 100 $\mathrm{cm}$ ) as within-participant factors. Greenhouse-Geisser corrections of degrees of freedom were used when sphericity assumptions were violated. For every statistically significant effect, we calculated effect sizes using generalized etasquared values (cf. Bakeman, 2005). These effect sizes were interpreted according to Cohen's (1988) recommendation of .02 for a small effect, .13 for a medium effect, and .26 for a large effect. In post-hoc analyses, we applied Bonferroni corrections to control Type-l errors.

\section{Results}

Fig. 1 shows typical examples of hand-aperture profiles. Figs. $1 A$ and $B$ present the hand aperture as a function of actual time to contact (i.e., the time until the end of the grasping movement), equivalent to the most familiar representation of hand apertures in prehension, and given in the majority of the studies of grasping and catching (e.g., in the context of prehension: Castiello, 2005; Jeannerod, 1984, 1988; Marteniuk, Leavitt, MacKenzie, \& Athènes, 1990; Zaal et al., 1998; but see Bongers, Zaal, \& Jeannerod, submitted; and in the context of catching: Mazyn, Savelsbergh, Montagne, \& Lenoir, 2007; Savelsbergh, Whiting, \& Bootsma, 1991; Savelsbergh, Whiting, Pijpers, \& van Santvoord, 1993). In Figs. 1C and D, hand aperture is plotted as a function of actual distance to contact (i.e., hand-object 
distance), a less familiar representation, although given in a subset of prehension studies that stress the role of distance rather than time (e.g., see Haggard \& Wing, 1998; Rand, Squire, \& Stelmach, 2006; Wallace, Stevenson, Spear, \& Weeks, 1994; Wing \& Fraser, 1983; Zaal \& Bootsma, 2000). Finally, Figs. 1E and F give hand aperture as a function of first-order time-to-contact $\left(T C_{1}(D)\right.$; cf. Lee 1976).

\subsection{Closing time, closing distance, and first-order time-to-contact}

Although we will perform our inferential statistics on the temporal prediction errors that we defined before, for the sake of comparison of the present data with results of previous papers, Table 2 gives the values of the three variables of closing time, closing distance, and $T C_{1}(D)$, at the moment of the initiation of hand closure. Table 2 presents the averages and average standard deviations of these three variables, for the three object-velocity conditions. As we explained before, we did not analyze these averages per se, but inspected temporal prediction errors, to allow a proper comparison among the variables (see Methods section for details).

${ }^{* * *}$ Fig. 1 and Table 2 about here ${ }^{* * *}$

We found a large effect of object velocity on the closing-time prediction error, $F(1.40,19.59)=28.77, p<.0001, \eta^{2} G=.269$ (see Table 3). Post-hoc tests learned that all means were different from each other $(p<.05)$. In addition, the ANOVA showed a large task effect, $F(1,14)=19.51, p<.0005, \eta_{G}^{2}=.432, M(S D)=12.1$ (22.0) and -11.6 (29.7) ms for catching and prehension, respectively.

The ANOVA on the closing-distance prediction error revealed a large effect of object velocity, $F(1.13,15.77)=119.42, p<.001, \eta_{G}^{2}=.503$ (see Table 3). The post-hoc tests showed that all levels of object velocity differed from each other $(p<$ 
.01). Furthermore, the ANOVA showed a small to medium Task x Object-velocity interaction, $F(1.32,18.49)=7.67, p<.05, \eta_{G}^{2}=.068$.

Finally, we found a small to medium effect of object velocity on the prediction error of first-order time-to-contact at the moment of hand-closure initiation, $F(1.21$, $16.91)=13.33, p<.005, \eta_{G}^{2}=.125$ (see Table 3). Post-hoc tests indicated that all differences between the means, except for the difference between the two highest object velocities, were statistically significant $(p<.05)$.

\section{${ }^{* * *}$ Table 3 about here ${ }^{* *}$}

\subsection{Dynamic-timing model}

As we mentioned in the Methods section, we first evaluated the model predictions using the same $\mathrm{c}_{\text {vision }}$ parameter setting for the prehension and the catching tasks. Inspection of the average prediction errors learned that in the catching task, the model, on average, was some $15.5(S D=23.4) \mathrm{ms}$ too late, whereas in the prehension task, it was some $8.5(S D=29.4)$ ms too early. Although these differences were rather small, a large task effect was found, $F(1$, 14) $=50.34, p<.001, \eta_{G}^{2}=.418$. Furthermore, we found a small to medium effect of object velocity, $F(1.25,17.53)=7.95, p<.01, \eta_{G}{ }_{G}=.117$ (see Table 3). Post-hoc tests showed that the average prediction error of the lowest object-velocity condition differed from the other object-velocity conditions $(p<.05)$. Finally, the ANOVA revealed a small to medium Task x Object-Velocity interaction, $F(1.19$, $16.67)=9.59, p<.01, \eta_{G}^{2}=.039$.

Given the two effects that include the factor of task, we were interested to see if allowing the $c_{\text {vision }}$ parameter to vary, not only across participants, but also across tasks, would result in a situation in which the model would predict the initiation of 
hand closure in both tasks accurately, albeit with different strengths of the optical information on the intrinsic dynamics of hand opening and closing. The ANOVA on the prediction errors revealed a same pattern of effects as we had found when we used the same values of the $c_{\text {vision }}$ parameter for both tasks, although with smaller effect sizes. The analysis showed a medium to large effect of object velocity, $F(1.23,17.28)=7.89, p<.01, \eta_{G}^{2}=.142$ (see Table 3$)$. Again, the prediction errors in the slowest object-velocity condition differed from those in the two other objectvelocity conditions $(p<.05)$. Furthermore, we found a small effect of task, $F(1,14)$ $=7.55, p<.05, \eta_{G}^{2}=.028, M(S D)=-3.6(22.9)$ and $1.1(29.5) \mathrm{ms}$ for catching and prehension, respectively. Finally, the ANOVA revealed a small Task x ObjectVelocity interaction effect, $F(1.24,17.42)=6.11, p<.05, \eta_{G}^{2}=.028$ (see Table 4). To unpack this interaction effect, we performed two ANOVAs, with factors of object velocity and initial distance, for the prehension task and catching task separately. Whereas we found no statistically significant effects of any of both factors for the prehension task, the analysis of the catching data revealed a large object-velocity effect, $F(1.22,17.13)=9.53, p<.010, \eta_{G}^{2}=.248$, due to different prediction errors when catching the objects that approached at the lowest velocity $(p<.05)$.

\section{*** Table 4 about here ${ }^{* * *}$}

\section{Discussion}

The main purpose of the current study was to see if the timing of closure of the hand was controlled similarly in prehension and catching, both with approaching objects. Given the earlier successes (Zaal \& Bootsma, 2004; Zaal et al., 1998) of modeling the initiation of hand closure in prehension with Schöner's (1994) dynamic timing model, with the hand-closure initiation timed on the basis of first- 
order time-to-contact information, we took this model as our starting point of the study. Zaal and Bootsma (2004) reported, for prehension of stationary objects, effects of distance, size, and width on the $T C_{1}(D)$ values in their experiment but that the dynamic timing model accommodated all these variations: They found no significant effects on the average prediction errors. The present study showed that this was not the case in all the conditions that we tested, in which we asked our participants to either reach for and grasp an object approaching at a constant velocity or catch it (with a stationary hand). We found effects of both object velocity and task on the quality of the dynamic timing model's prediction of the moment of hand-closure initiation. Importantly, we also found an interaction effect of these two factors. It turned out that dynamic timing model had difficulty fitting particularly the condition of catching the object approaching at its lowest velocity; In the other conditions, the prediction errors were less than $5 \mathrm{~ms}$, on average.

In line with previous studies, which had shown that closing time (Laurent et al., 1994; Rand \& Stelmach, 2005; Rand et al., 2006, 2008; Zaal \& Bootsma, 2004) and closing distance (e.g., Carnahan \& McFadyen, 1996; Rand \& Stelmach, 2005; Rand et al., 2006; Wang \& Stelmach, 2001; Watson \& Jakobson, 1997; Zaal \& Bootsma, 2004; Zaal et al., 1998) varied with factors such as object velocity, object orientation, object width, object size, reaching amplitude, and reaching velocity, we found a large effect of the velocity with which the object approached on these two variables. The effects of object velocity on these two factors that came out of our experiment corroborated the conclusion that closing time or distance are not being kept constant, and, thus, do not act as control variables in the coordination of reaching and grasping or in the timing of catching in the situation that the hand is not moving. 
Our results showed that the dynamic timing model did not perform well for the current catching task of a stationary hand and an object approaching at constant velocity, especially when the target arrived at a low speed. From this, one might conclude that the model was not appropriate to explain the timing of hand-closure initiation in catching. An alternative conclusion might be that our choice of designing the catching task in such a constrained way might have brought our participants in a rather unnatural situation, in which they were invited to show behavior that they would not show in natural catching. We have planned experiments to explore this possibility. In these experiments, objects arrive at nonconstant velocities or we allow the hand to move when objects do arrive at constant velocities.

When we looked at the different object-velocity effects in our data, we found that the dynamic timing model most closely fit the patterns of results of the current experiment. Except for the condition of catching approaching objects at the lowest speed used in the current study, temporal prediction errors were less than $5 \mathrm{~ms}$ (cf. Table 4). The dynamic timing model did explain the results just a little better than a model with the timing of the initiation of hand closure at a critical value of first-order time-to-contact, and much better than models in which closure distance or time were to be kept constant. Temporal prediction errors of the constant-time-to-contact account were roughly twice as big as those of the dynamic timing model, except for the prediction errors of the lowest object velocity (see Table 3). This lowest object velocity proved to be problematic, particularly for the current catching task (we did not find a significant object-velocity effect when we considered the data of the prehension task separately). Taken together, the dynamic timing model accounted for much of the variability seen in closing time and closing distance, and a small amount of the variability seen in first-order time-to-contact. For now, we conclude 
that the dynamic timing model does a fine, albeit not a perfect, job in predicting the moment of the initiation of hand closure on the basis of first-order time-to-contact, at least for the task of prehension. As to the task of catching, the jury is still out. 


\section{Acknowledgment}

This research was supported by a VIDI grant (452-03-356) from the Netherlands Organization for Scientific Research (NWO) awarded to Frank Zaal. We thank

Henry van de Crommert, Wim Kaan, and Wolter de Goede for developing the setup that we used in the study, and two anonymous reviewers for their helpful comments on an earlier draft of the current paper. 


\section{Footnote}

${ }^{1}$ Following Bootsma, Fayt, Zaal, and Laurent (1997), we distinguish the optical time-to-contact information $(\tau)$ from the organism-environment property that it specifies, $T C_{1}(D) . T C_{1}(D)$ is defined as the first-order time-to-contact, the time that distance gap $D$ will be closed when closing velocity would remain constant. Although our ultimately interest is in the information, technically speaking, it is the physical first-order time-to-contact $T C_{1}(D)$ that we manipulated in the current study, and that we used in our model simulations. 
Getting hold of approaching objects -- 21

\section{References}

Bakeman, R. (2005). Recommended effect size statistics for repeated measures designs. Behavior Research Methods, 37, 379-384.

Bennett, S. J., van der Kamp, J., Savelsbergh, G. J. P., \& Davids, K. (1999). Timing a one-handed catch I. Effects of telestereoscopic viewing. Experimental Brain Research, 129, 362-368.

Bongers, R. M., Zaal, F. T. J. M., \& Jeannerod, M. (submitted). Hand aperture patterns in prehension.

Bootsma, R. J., Fayt, V., Zaal, F. T. J. M., \& Laurent, M. (1997). On the informationbased regulation of movement: What Wann (1996) may want to consider. Journal of Experimental Psychology: Human Perception and Performance, 23, 1282-1289.

Bootsma, R. J., \& Peper, C. E. (1992). Predictive visual information sources for the regulation of action with special emphasis on catching and hitting. In L. Proteau \& D. Elliott (Eds.), Vision and motor control (pp. 285-314). Amsterdam: NorthHolland.

Caljouw, S. R., van der Kamp J., \& Savelsbergh, G. J. P. (2004). Catching optical information for the regulation of timing. Experimental Brain Research, 155, 427438.

Carnahan, H., \& McFadyen, B. J. (1996). Visuomotor control when reaching toward and grasping moving targets. Acta Psychologica, 92,17-32.

Castiello, U. (2005). The neuroscience of grasping. Nature Reviews Neuroscience, 6, 726-736.

Cohen, J. (1988). Statistical power analysis for the behavioral sciences (2nd ed.). Hillsdale, NJ: Erlbaum. 
Gentilucci, M., Chieffi, S., Scarpa, M., \& Castiello, U. (1992). Temporal coupling between transport and grasp components during prehension movements: effects of visual perturbation. Behavioral Brain Research, 47, 71-82.

Haggard, P. \& Wing, A. (1998). Coordination of hand aperture with the spatial path of hand transport. Experimental Brain Research, 118, 286-292.

Jeannerod, M. (1984). The timing of natural prehension movements. Journal of Motor Behavior, 16, 235-254.

Jeannerod, M. (1988). The neural and behavioural organization of goal-directed movements. Oxford, UK: Oxford University Press.

Laurent, M., Montagne, G., \& Savelsbergh, G. J. P. (1994). The control and coordination of one-handed catching: The effect of temporal constraints. Experimental Brain Research, 101, 314-322.

Lee, D. N. (1976). A theory of visual control of braking based on information about time-to-collision. Perception, 5, 437-459.

Marteniuk, R. G., Leavitt, J. L., MacKenzie, C. L., \& Athènes, S. (1990). Functional relationships between grasp and transport components in a prehension task. Human Movement Science, 9, 149-176.

Mazyn, L. I. N., Montagne, G., Savelsbergh, G. J. P., \& Lenoir, M. (2006).

Reorganization of catching coordination under varying temporal constraints. Motor Control, 10, 143-159.

Mazyn, L. I. N., Savelsbergh, G. J. P., Montagne, G., \& Lenoir, M. (2007). Planning and on-line control of catching as a function of perceptual-motor constraints. Acta Psychologica, 126, 59-78.

Rand, M. K., \& Stelmach, G. E. (2005). Effect of orienting the finger opposition space in the control of reach-to-grasp. Journal of Motor Behavior, 37, 65-78. 
Rand, M. K., Squire, L. M., \& Stelmach, G. E. (2006). Effect of speed manipulation on the control of aperture closure during reach-to grasp movements. Experimental Brain Research, 174, 74-85.

Rand, M. K., Shimansky, Y. P., Hossain, A. B. M. I., \& Stelmach, G. E. (2008). Quantitative model of transport-aperture coordination during reach-to-grasp movements. Experimental Brain Research, 188, 263-274.

Savelsbergh, G. J. P., Whiting, H. T. A., \& Bootsma, R. J. (1991). Grasping tau. Journal of Experimental Psychology: Human Perception and Performance, 17, 315-322.

Savelsbergh, G. J. P., Whiting, H. T. A., Burden, A. M., \& Bartlett, R. M. (1992). The role of predictive visual temporal information in the coordination of muscle activity in catching. Experimental Brain Research, 89, 223-228.

Savelsbergh, G. J. P., Whiting, H. T. A., Pijpers, J. R., \& van Santvoord, A. A. M. (1993). The visual guidance of catching. Experimental Brain Research, 93, 148156.

Schenk, T., Philipp, J., Haussler, A., Hauck, A., Hermsdorfer, J., \& Mai, N. (2000). A system for the study of visuomotor coordination during reaching for moving targets. Journal of Neuroscience Methods, 100, 3-12.

Schöner, G. (1994). Dynamic theory of action-perception patterns: The time-beforecontact paradigm. Human Movement Science, 13, 415-439.

Wallace, S. A., Stevenson, E., Weeks, D. L., \& Kelso, J. A. S. (1992). The perceptual guidance of grasping a moving object. Human Movement Science, 11, 691-715.

Wang, J. S., \& Stelmach, G. E. (1998). Coordination among the body segments during reach-to-grasp action involving the trunk. Experimental Brain Research, 123, 346-350. 
Wang, J. S., \& Stelmach, G. E. (2001). Spatial and temporal control of trunkassisted prehensile actions. Experimental Brain Research, 136, 231-240.

Watson, M. K., \& Jakobson, L. S. (1997). Time to contact and the control of manual prehension. Experimental Brain Research, 117, 273-280.

Wing, A. M. \& Fraser, C. (1983). The contribution of the thumb to reaching movements. Quarterly Journal of Experimental Psychology A, 35, 297-309.

Zaal, F. T. J. M., \& Bootsma, R. J. (2004). The use of time-to-contact information for the initiation of hand closure in natural prehension. In H. Hecht \& G. J. P. Savelsbergh (Eds.), Time-to-contact (pp. 389-420). Amsterdam: North-Holland.

Zaal, F. T. J. M., Bootsma, R. J., \& van Wieringen, P. C. W. (1998). Coordination in prehension: Information-based coupling of reaching and grasping. Experimental Brain Research, 119, 427-435. 


\section{Appendix: Model equations}

The dynamic timing model is a set of differential equations, originally formulated by Schöner (1994), and adopted for the situation of the grasping of prehension by Zaal and colleagues (Zaal \& Bootsma, 2004; Zaal et al., 1998). In the model, a state variable $x$ is mapped onto the hand-opening and hand-closing regimes of prehension. The model equations combine so-called intrinsic dynamics -

- the dynamics that give the state variable $x$ its stability properties -- and the contribution of the visual information:

$$
\frac{d}{d t}\left(\begin{array}{l}
x \\
y
\end{array}\right)=f_{\text {grasp }}+f_{\text {vision }}
$$

In the model three attractors in state space are defined. That is to say, there are two fixed-point attractors, for the hand-opening regime $\left(x_{o p e n}\right)$ and the hand-closing regime $\left(x_{\text {close }}\right)$, respectively, and a limit-cycle attractor passing through these two fixed-point attractors:

$$
f_{\text {grasp }}=f_{\text {osc }}+f_{\text {open }}+f_{\text {close }}
$$

$$
\begin{aligned}
& f_{\text {osc }}(x, y)=\left(\begin{array}{cc}
\alpha & \omega \\
-\omega & \alpha
\end{array}\right)\left(\begin{array}{l}
x \\
y
\end{array}\right)-\gamma\left(\begin{array}{l}
\left(x^{2}+y^{2}\right) x \\
\left(x^{2}+y^{2}\right) y
\end{array}\right) \\
& f_{\text {open }}(x, y)=-\beta_{\text {int }} f_{\text {range }}\left(\begin{array}{l}
x-x_{\text {open }} \\
y-y_{\text {open }}
\end{array}\right)
\end{aligned}
$$




$$
\begin{aligned}
& f_{\text {close }}(x, y)=-\beta_{\text {int }} f_{\text {range }}\left(\begin{array}{l}
x-x_{\text {close }} \\
y-y_{\text {close }}
\end{array}\right) \\
& f_{\text {range }}\left(x, y, x_{i}, y_{i}\right)=\exp \left[-\frac{\left(x-x_{i}\right)+\left(y-y_{i t}\right)}{2 \sigma^{2}}\right]
\end{aligned}
$$

The contribution of the visual variable $r(D)$, which is the inverse of $T C_{1}(D)$ in our case, is defined:

$$
\begin{aligned}
& f_{\text {vision }}\left(x, y, x_{\text {open }}, y_{\text {open }}, x_{\text {close }}, y_{\text {close }}\right)=\beta_{\text {vision }}(D) \mid+f_{\text {range }}\left(x, y, x_{\text {open }}, y_{\text {open }}\right)\left(\begin{array}{l}
x-x_{\text {open }} \\
y-y_{\text {open }}
\end{array}\right) \\
& \left.-f_{\text {range }}\left(x, y, x_{\text {close }}, y_{\text {close }}\right)\left(\begin{array}{l}
x-x_{\text {close }} \\
y-y_{\text {close }}
\end{array}\right)\right] \\
& \beta_{\text {vision }}(D)=c_{\text {vision }}\left(r(D)-r_{\text {crit }}\right)
\end{aligned}
$$

A closer inspection of Equation (3a) shows large similarities with Equations (2c) and (2d). A growing value of the visual variable $r(D)$ leads to an increase of the

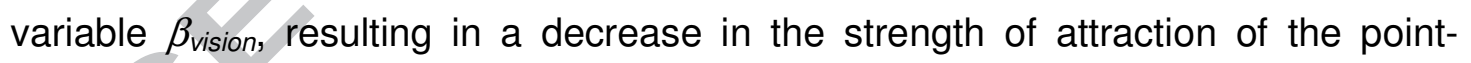
attractor at $x_{\text {open }}$ and an increase in the strength of attraction of the point attractor at $x_{\text {close }}$. For a more detailed introduction to the model and its equations, we refer the reader to Schöner (1994). 


\section{Table 1}

Values of the $c_{\text {usion }}$ parameter that were used in the simulations when the same parameter was used for both tasks and when different parameters were used for the two tasks of catching and prehension.

\begin{tabular}{llll}
\hline participant & \multicolumn{3}{l}{$C_{\text {vison }}$ parameter } \\
\cline { 2 - 4 } & same & different & \\
\cline { 2 - 4 } & & catching & prehension \\
\hline 1 & 4.21 & 5.29 & 3.90 \\
2 & 4.27 & 4.23 & 4.27 \\
3 & 4.67 & 4.85 & 4.59 \\
4 & 4.37 & 5.37 & 4.14 \\
5 & 3.75 & 4.82 & 3.62 \\
6 & 4.32 & 4.70 & 4.18 \\
7 & 3.87 & 4.72 & 3.63 \\
8 & 3.96 & 5.89 & 3.59 \\
9 & 3.86 & 4.95 & 3.53 \\
10 & 6.04 & 6.10 & 5.80 \\
11 & 4.40 & 4.57 & 3.86 \\
12 & 4.11 & 4.14 & 3.97 \\
13 & 4.89 & 5.00 & 4.89 \\
14 & 3.50 & 3.85 & 3.23 \\
15 & 3.80 & 4.64 & 3.73 \\
\hline
\end{tabular}




\section{Table 2}

Means and average within-participant standard deviations (between brackets) of closing time, closing distance, and first-order time-to-contact $T C_{1}(D)$, at the moment of initiation of hand closure, as a function of object velocity.

\begin{tabular}{llll}
\hline & \multicolumn{3}{l}{ object velocity $(\mathrm{cm} / \mathrm{s})$} \\
\cline { 2 - 4 } & 20 & 40 & 60 \\
\hline Closing Time $(\mathrm{ms})$ & $122.4(32.0)$ & $107.9(24.8)$ & $100.1(20.7)$ \\
Closing Distance $(\mathrm{mm})$ & $12.5(6.6)$ & $18.0(6.7)$ & $25.3(8.2)$ \\
$T C_{1}(D)(\mathrm{ms})$ & $54.8(16.0)$ & $47.9(11.5)$ & $46.3(10.5)$ \\
\hline
\end{tabular}




\section{Table 3}

Means and average within-participant standard deviations (between brackets) of the model prediction errors (ms) as a function of object velocity.

\begin{tabular}{|c|c|c|c|}
\hline & \multicolumn{3}{|c|}{ object velocity $(\mathrm{cm} / \mathrm{s})$} \\
\hline & 20 & 40 & 60 \\
\hline Constant closing time & $-11.0(32.0)$ & $3.5(24.8)$ & $3.3(20.7)$ \\
\hline Constant closing distance & $12.1(22.0)$ & $-7.7(14.4)$ & $-18.4(12.9)$ \\
\hline Constant first-order time-to-contact & $-2.7(25.8)$ & $6.1(16.1)$ & $7.7(14.3)$ \\
\hline Dynamic timing & $-10.8(35.2)$ & $0.7(21.6)$ & $-0.5(22.4)$ \\
\hline Different $\mathrm{c}_{\text {vision }}$ & $-8.4(34.9)$ & $3.0(21.7)$ & $2.2(22.1)$ \\
\hline
\end{tabular}




\section{Table 4}

The significant Object-Velocity $x$ Task interaction effect of the dynamic-timingmodel prediction errors (ms); Means and average within-participant standard deviations (between brackets).

\begin{tabular}{llll}
\hline Task & \multicolumn{3}{l}{ object velocity $(\mathrm{cm} / \mathrm{s})$} \\
\cline { 2 - 4 } & 20 & 40 & 60 \\
\hline Prehension & $-3.2(36.3)$ & $3.6(26.2)$ & $2.8(26.1)$ \\
Catching & $-13.7(33.5)$ & $2.4(17.2)$ & $1.5(18.1)$ \\
\hline
\end{tabular}


Getting hold of approaching objects -- 31

\section{Figure caption}

Fig. 1. Typical examples of hand-aperture profiles of 6 different trials of one and the same participant, of the catching task (left column) and of the prehension task (right column). The dotted, dashed, and solid lines represent the 20,40, and 60 $\mathrm{cm} / \mathrm{s}$ levels of object velocity, respectively. The hand-aperture profiles are plotted as a function of the actual time to contact (top row), the actual distance to contact (middle row), and the first-order time-to-contact (bottom row). 


\section{Catching}
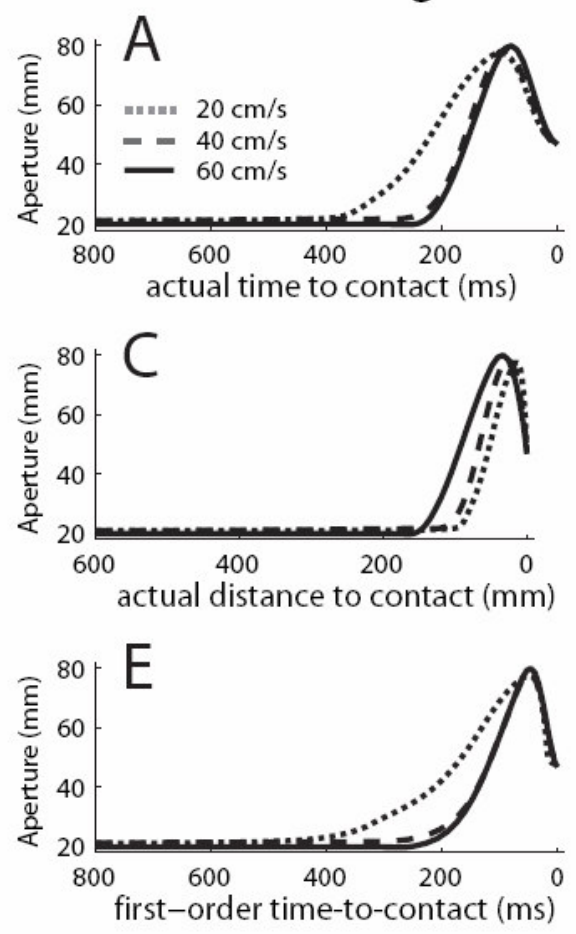

Prehension
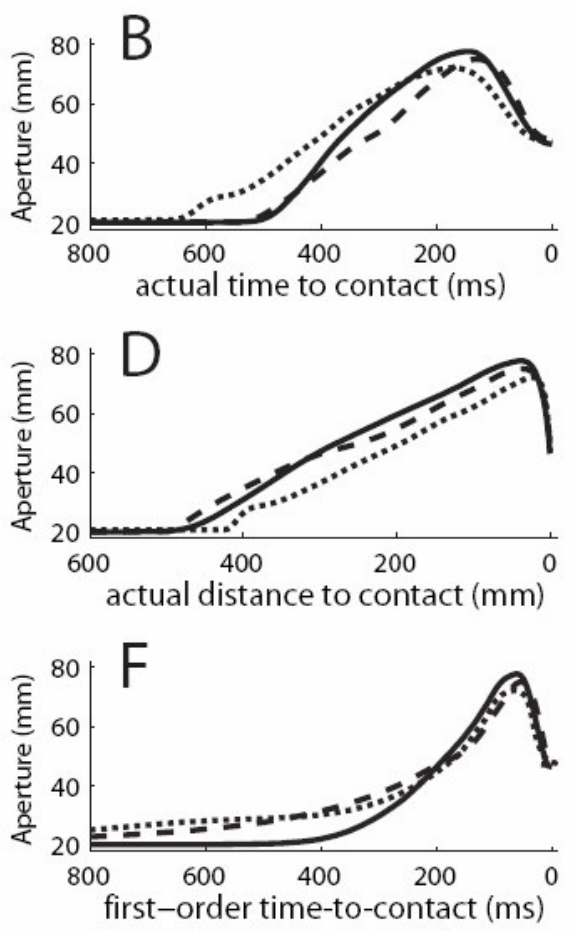

Fig .1 